\title{
Effects of strain, sex and age on immunophenotyping parameters in the rat and mouse
}

\author{
Katie Ridge $^{1} \cdot$ Noel Downes $^{1} \cdot$ Brenda Finney $^{1}$ \\ Received: 10 January 2018 / Accepted: 22 March 2018 / Published online: 13 April 2018 \\ (C) The Author(s) 2018
}

\begin{abstract}
Immunophenotyping of lymphocyte subsets can be a useful addition to toxicological or investigatory studies where there is the potential for effects on the immune system. We have validated assays for rat or mouse lymphocytes using a FACSVerse flow cytometer and FACSuite software, with commercially available antibodies. Whole blood was stained using the following markers: Rat-CD3, CD45RA, CD161a, CD4 and CD8; mouse-CD3e, CD19, NK1.1, CD4 and CD8. This allows detection and quantitation of T cells, B cells, NK cells, T helper cells and T cytotoxic cells. The stopping criteria for analysis were set at 30,000 lymphocytes, although data were considered acceptable if a minimum of 5000 lymphocytes were counted. To maintain the same analysis protocol throughout and across studies, an assay was created for each species in the FACSuite software. Assay functionality uses CS\&T beads for performance quality control and assay setup to ensure that laser settings remain consistent and compensation settings are such that any drift in machine performance does not affect results. Blood samples from both sexes and the following ages and strains were analysed: Rats $-2,4$ and 7 months, $\mathrm{Crl}$ :WI(Han) and 2 months $\mathrm{Crl}: \mathrm{CD}(\mathrm{SD})$; mice $-2-3,4-6$ and 7-8 months, Crl:CD1(ICR). In general, there was a low level of variation within the results for each sex or age group. However, there are strain, sex and age differences that should be considered when evaluating results from immunophenotyping analysis or when planning studies where there may be an immunological effect.
\end{abstract}

Keywords Immunophenotyping $\cdot$ Flow cytometry $\cdot$ Rat $\cdot$ Mouse $\cdot$ Strain effects

\section{Introduction}

Immunophenotyping uses specific antibodies for each cell of interest to identify specific leukocyte subsets. Although it can be conducted using immunohistochemistry, it is increasingly being performed by flow cytometry in order to obtain quantitative data. While immunohistochemistry offers its own advantages, an assay using commercially available, specific, fluorescently tagged antibodies allows for the direct interrogation of a blood sample by multicolour flow cytometry. Multiple cell types can be identified and quantified in a single blood sample, helping to limit sample volume, which is particularly useful in the rodent species routinely used for preclinical toxicology. By immunophenotyping peripheral blood, data on specific components of the white blood cell (WBC)

Brenda Finney

brenda.finney@sequani.com

1 Sequani Limited, Bromyard Road, Ledbury, Hereford HR8 1LH, UK population can be generated to complement and enhance the data collected in routine haematology analysis. It can also contribute to the translation of the effects in animals to humans as the ICH S8 guideline on immunotoxicity studies for human pharmaceuticals state, "Data obtained from peripheral blood can be useful as a bridge for clinical studies in which peripheral blood leukocytes are also evaluated." (ICH 2005).

Immunophenotyping is recommended in pre-clinical studies when there is the potential for adverse effects on the immune system by pharmaceuticals and is generally used within the context of a wider immunotoxicity investigation. For reference, immunotoxicity is the unintended enhancement or suppression of the immune system (ICH 2005) and the conduct of this investigation is based on the weight of evidence for the compound which will also influence the species and strain used for testing. The assays used in this paper were setup on the FACSVerse flow cytometer using FACSuite software. The functionality of this software allows for assay-based function and uses CS\&T beads for performance quality control and assay setup to ensure that laser settings remain within a specific range and compensation settings are correct for each fluorophore used. These 
compensation settings are such that any drift in machine performance does not affect results over time, ensuring that the same analysis protocol is performed throughout all studies, which is an important aspect of validation within a GLP-compliant facility and aids in the evaluation of data for regulatory purposes. The assay validation was based on recommendations in partner publications by Green et al. (2011) and O'Hara et al. (2011) in the Journal of Immunological Methods.

For the detection and quantitation of T cells, B cells, NK cells, $\mathrm{T}$ helper cells and $\mathrm{T}$ cytotoxic cells, whole blood was stained using markers relevant for each species. In the rat, CD3, CD45RA, CD161a, CD4 and CD8a were used. In the mouse, CD3e, CD19, NK1.1, CD4 and CD8 were used. Blood samples from both sexes and the following ages and strains were analysed: Rats 2 months $\mathrm{Crl}: \mathrm{CD}(\mathrm{SD}), 2,4$ and 7 months Crl:WI(Han); mice 2-3, 4-6 and 7-8 months, Crl:CD-1(ICR). The objective of this study was to compare and contrast data across strains, sexes and ages, in order to understand the variation of these factors when choosing experimental subjects. The data contained in this paper are also used as historical control data, for comparison to data collected during regulatory toxicological investigations. It is hoped that the protocols and data presented in this paper will be of use to other laboratories that are performing or looking to perform this type of assay on these species and strains.

Difference in gating strategies between analysts, analysers and laboratories is a primary reason why flow cytometry data sets are difficult to compare. To control for this in our laboratory, we have used the assay functionality of the FACSuite software in conjunction with the FACSVerse flow cytometer. This function ensures that when gates are set and saved as part of the assay, the gates are in the same position for each sample each time the assay is opened. In terms of GLP compliance, it also ensures that the initial settings of the assay can be compared with any data used for reporting, and changes can be detected or annotated between samples or days of analysis. As a part of the assay function, compensation settings are routinely checked and updated using quality control beads. These beads are either fluorophore-specific, Research FC beads or wide-spectrum CS\&T beads. There have been initial steps taken to standardise or automate gating strategies across institutions and platforms, but as of yet, these are not widely used.

Due to the use of mice in immunology research, there appears to be a greater amount of published material on the immunophenotype found in mouse strains than in rat strains. However, data on the Crl:CD1(ICR) mouse strain were not found, nor were there data comparing different ages. Although published data on rat strains were limited, information was found on immunophenotyping in RccHan:Wist (Yamatoya et al. 2012) and Sprague Dawley (Morris and Komocsar, 1997). This paper expands the available strain data and provides a direct comparison of results between two rat strains and shows that it is worth considering age, sex and strain differences when planning a study or evaluating the data produced from an investigation.

\section{Materials and methods}

All samples were analysed on a FACSVerse flow cytometer using FACSuite software (BD Biosciences, UK). After initial setup, an assay was created with specific tube settings and gates relevant to each assay. This ensured that all sample analysis was done to the same specifications. Tube and assay settings were set using Research FC beads (BD Biosciences, UK). Cytometer setup and quality control were performed on each day of cytometer use according to the manufacturer's instructions using CS\&T beads. Due to the ability to use CS\&T and Research FC beads for the setup and maintenance of the compensation settings needed for each assay, isotype controls were not used. However, depending upon the analyser used, isotype controls may be required when establishing this assay in other laboratories. Additionally, Comp beads, with positive and negative populations, can be used to determine parameters for the specific antibodies used in each assay. Specific consumables purchased from BD Biosciences, UK, have specific information provided in Table 1.

\section{Methods}

\section{Use of animals}

All institutional and national guidelines for the care and use of laboratory animals were followed and all animal work was conducted under authority of a Project Licence in compliance with the Animals (Scientific Procedures) Act 1986 (as amended). Euthanasia and blood collection were done according to routine standard operating procedures.

\section{Blood sampling and sample processing}

Blood was collected from either the sublingual vein (rats only), abdominal vena cava (both species) or orbital sinus (mice only) into tubes containing $\mathrm{K}_{2}$ EDTA depending upon the study type from which the animals were taken. The tubes were then mixed on a roller at room temperature for 5-10 $\mathrm{min}$. After mixing, they were kept on ice or at $2-8{ }^{\circ} \mathrm{C}$ until processed. Processing was performed on the day of collection. Cell counts were performed on an ADVIA 120 (Siemens, UK) haematology analyser and samples were normalised to $1 \times$ $10^{6}$ lymphocytes $/ \mathrm{mL}$ with phosphate buffered saline (PBS). After normalisation, antibodies were added to the sample at the concentrations listed in Table 1. The samples were incubated in the dark at room temperature. The incubation was ended by the addition of $20 \times$ volume of FACSLyse and vortexed. Samples were then centrifuged at $500 \mathrm{~g}$ for $5 \mathrm{~min}$, 
Table 1 Reagents and consumables used for immunophenotyping assays

\begin{tabular}{|c|c|c|c|c|}
\hline Reagent & $\begin{array}{l}\text { Catalogue } \\
\text { number }\end{array}$ & $\begin{array}{l}\text { Assay } \\
\text { species }\end{array}$ & Cell types and fluorophore & Amount used per test (if applicable) \\
\hline CS\&T beads & 650621 & $\mathrm{~N} / \mathrm{A}$ & N/A & $\mathrm{N} / \mathrm{A}$ \\
\hline FACSLyse & 349202 & $\mathrm{~N} / \mathrm{A}$ & $\mathrm{N} / \mathrm{A}$ & $20 \times$ sample volume \\
\hline $\mathrm{T}, \mathrm{B}, \mathrm{NK}$ cell cocktail & 558495 & Rat & $\begin{array}{l}\text { T cell: CD3, APC } \\
\text { B cell: CD45RA, FITC } \\
\text { NK cell: CD161a, PE }\end{array}$ & $10 / 100 \mu \mathrm{L}$ sample at $1 \times 10^{6}$ lymphocytes $/ \mathrm{mL}$ \\
\hline $\begin{array}{l}\text { CD45 antibody } \\
\text { (Used during initial assay setup, information } \\
\text { included for reference) }\end{array}$ & $\begin{array}{l}561586 \\
557659\end{array}$ & $\begin{array}{l}\text { Rat } \\
\text { Mouse }\end{array}$ & Lymphocytes, APC-Cy7 & $\begin{array}{l}2 / 100 \mu \mathrm{L} \text { (rat) } 200 \mu \mathrm{L} \text { (mouse) sample at } 1 \times 10^{6} \\
\text { lymphocytes } / \mathrm{mL}\end{array}$ \\
\hline T lymphocyte cell cocktail & 558493 & Rat & $\begin{array}{l}\text { T cell: CD3, APC } \\
\text { T helper cell: CD4, PE } \\
\text { T cytotoxic cell: CD8a, FITC }\end{array}$ & $10 / 200 \mu \mathrm{L}$ sample at $1 \times 10^{6}$ lymphocytes $/ \mathrm{mL}$ \\
\hline T lymphocyte cell cocktail & 558431 & Mouse & $\begin{array}{l}\text { T cell: } \mathrm{CD} 3 \mathrm{e}, \mathrm{PE}-\mathrm{Cy} 7 \\
\text { T helper cell: } \mathrm{CD} 4, \mathrm{PE} \\
\text { T cytotoxic cell: } \mathrm{CD} 8, \mathrm{APC}\end{array}$ & $10 / 200 \mu \mathrm{L}$ sample at $1 \times 10^{6}$ lymphocytes $/ \mathrm{mL}$ \\
\hline CD19 antibody & 557398 & Mouse & B cell: FITC & $2 / 200 \mu \mathrm{L}$ sample at $1 \times 10^{6}$ lymphocytes $/ \mathrm{mL}$ \\
\hline NK1.1 antibody & 560618 & Mouse & NK cell: APC-Cy7 & $2 / 200 \mu \mathrm{L}$ sample at $1 \times 10^{6}$ lymphocytes $/ \mathrm{mL}$ \\
\hline
\end{tabular}

most of the supernatant removed and $2 \mathrm{~mL}$ PBS added. The cells were resuspended by tapping the tube and then centrifuged at $500 \mathrm{~g}$ for $5 \mathrm{~min}$. The supernatant was then removed and the cells resuspended in $300 \mu \mathrm{L}$ PBS for analysis.

\section{Rat immunophenotyping}

Data were collected from animals of both sexes in the Crl:WI(Han) and Crl:CD(SD) strains (Charles River, UK). The age of the animals varied by strain. $\mathrm{Crl}: \mathrm{CD}(\mathrm{SD})$ data were collected at 2 months of age while $\mathrm{Crl}$ :WI(Han) data were obtained at 2, 4 and 7 months of age. Comparisons between strains were made on animals of the same sex and similar ages.

This assay was performed as a two-tube assay which was dictated by the fluorophores available in the staining cocktails. One tube was the lymphocyte assay which counted T, B and NK cells; the second tube counted T cells, CD4 + T helper cells and CD8 $+\mathrm{T}$ cytotoxic cells using the staining cocktails in Table 1. In the T, B and NK cell assay, samples were analysed until 30,000 cells in the lymphocyte gate were counted. Samples were accepted if at least 5000 lymphocytes were counted. In the T cell subset, tube samples were analysed until $5000 \mathrm{~T}$ cells were counted and accepted if at least $2500 \mathrm{~T}$ cells were counted. Samples were checked for stability with both of the staining cocktails. Sample results were acceptable only on the day following collection where samples had been stained, lysed and washed prior to storage at $2-8^{\circ} \mathrm{C}$.

The gating strategies for these assays are shown in Figs. 1 and 2. The initial assay setup utilised the pan-lymphocyte marker CD45 labelled with APC-Cy7 to confirm the establishment of the lymphocyte population gate. The lymphocyte cluster was initially identified based on FSC and SSC characteristics; when labelled with CD45, the constituents of this gate showed fluorescence in the $10^{4}-10^{5}$ region on a log scale. The lymphocyte population gate in the FSC vs. SSC dot plot was used as the gate for the stopping criteria of 30,000 lymphocytes. As FSC and SSC characteristics should not change greatly between animals, the use of the CD45 marker was discontinued after several samples confirmed the location of the gate on the FSC vs. SSC plot. Sub-population gates were also confirmed by plots comparing the different CD markers used to each other to check for overlap.

\section{Mouse immunophenotyping}

Data were collected from both sexes in the Crl:CD1(ICR) strain (Charles River, UK) at 2-3, 4-6 and 7-8 months of age.

This was a single-tube assay for all cell types. Samples were analysed until 30,000 cells in the lymphocyte gate were counted. Samples were accepted if at least 5000 lymphocytes were counted. Stability was tested during the validation; however, changes in cell populations in the conditions tested on subsequent days required that samples be analysed on the same day. The gating strategy for this assay is shown in Fig. 3.

\section{Data and statistics}

The data collected included both the absolute cell counts and the percentage of the cell subtypes present in the parent populations, either lymphocytes or T cells depending on the cell types. Data were correlated in a Microsoft Excel spreadsheet before transfer to GraphPad Prism (GraphPad Software, CA, USA), which was used for graph production and statistical analysis. T helper:T cytotoxic ratio for 2-month rat samples 

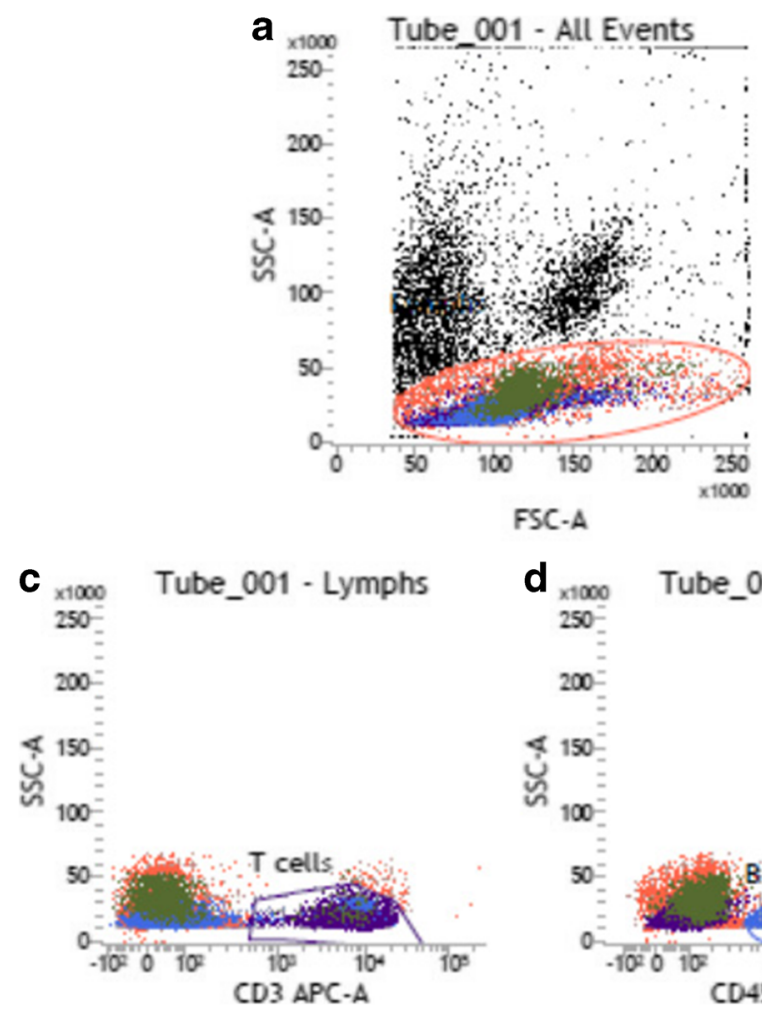

b

$-A$
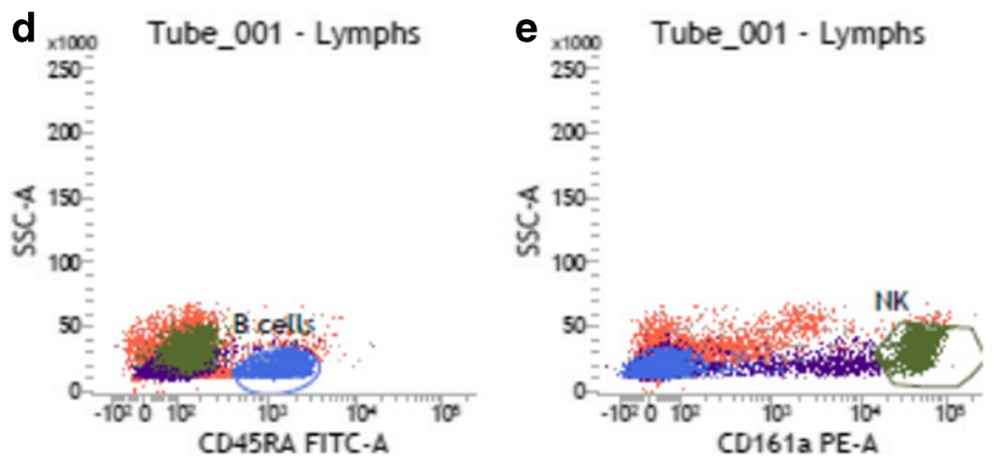

Fig. 1 Gating strategy in rat T, B and NK cell assay. a FSC vs. SSC graph, orange circle highlights the lymphocyte population. b Gating hierarchy for assay. $\mathbf{c}$ Purple gate indicating T cell population based on

expression of CD3. d Blue gate indicating $\mathrm{B}$ cell population based on CD45RA expression. e Green gate indicating NK cell population based on CD161a expression
Fig. 2 Gating strategy in rat T cell subset assay. a Gating hierarchy for assay. b Purple T cell gate based on $\mathrm{CD} 3$ expression. $\mathbf{c}$ Green circle gate for $\mathrm{T}$ helper cell population based on CD4 expression. $\mathbf{d}$ Red circle gate for $\mathrm{T}$ cytotoxic cells based on CD8 expression a
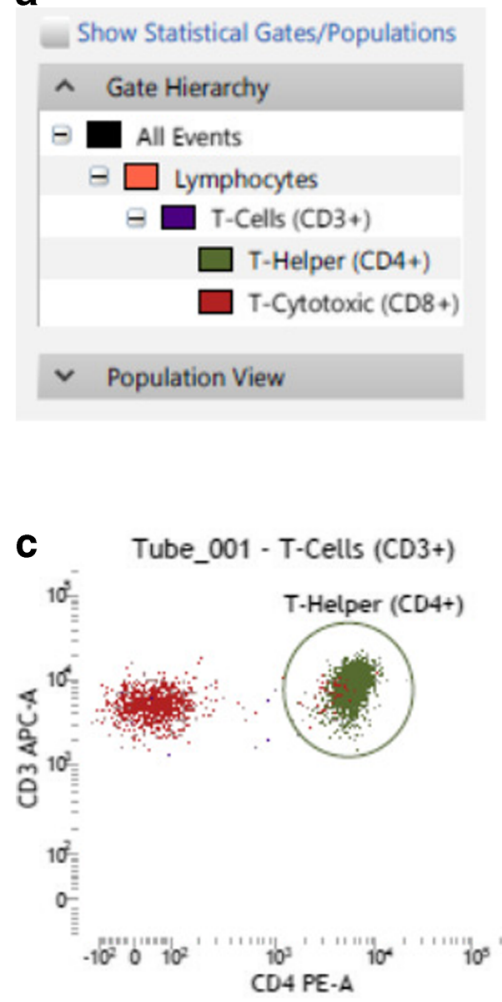

b
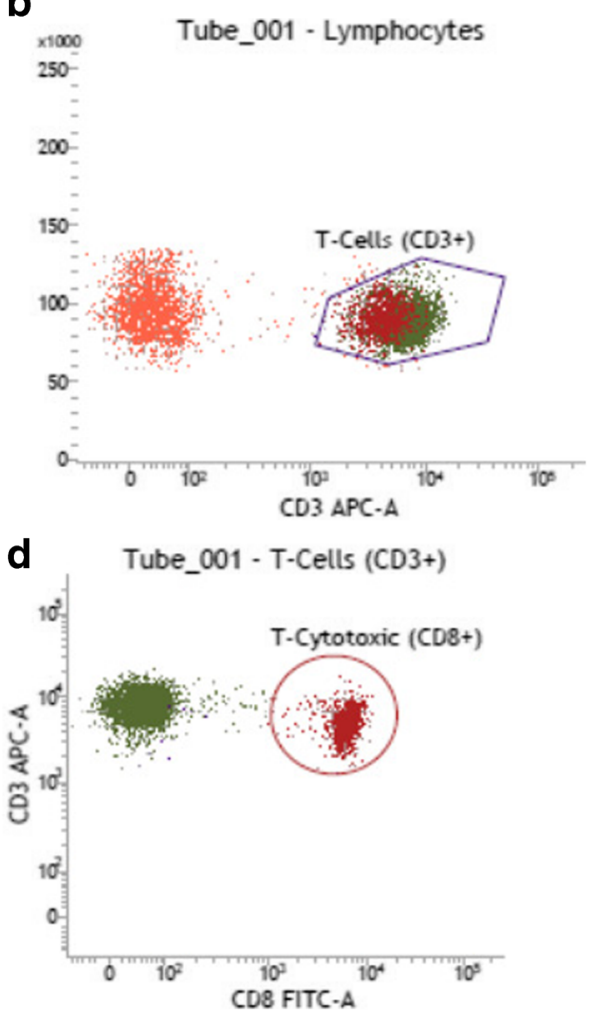

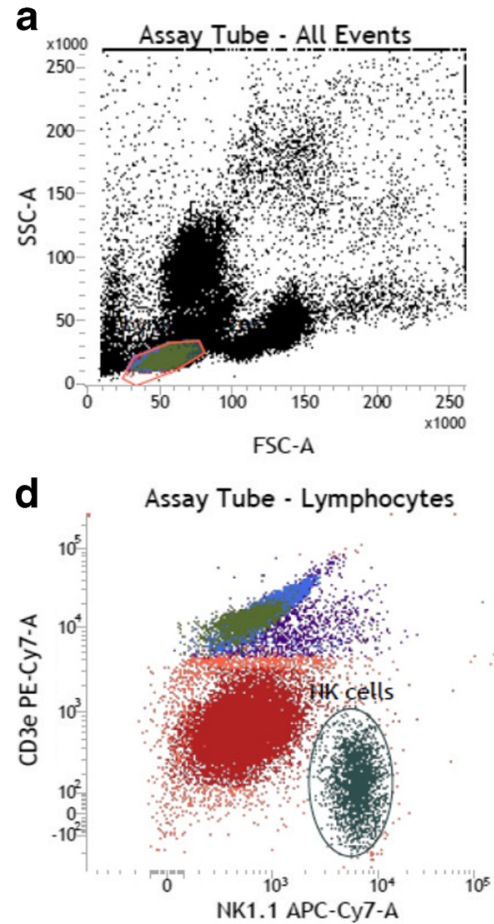

Fig. 3 Gating strategy for mouse immunophenotyping assay. a FSC vs. SSC graph, orange circle highlights the lymphocyte population. b Purple gate indicating T cell population based on expression of CD3e. $\mathbf{c}$ Red gate indicating B cell population based on CD19 expression. $\mathbf{d}$ Teal circle gate

was calculated by dividing the absolute $\mathrm{T}$ helper cell number (CD4+) by the absolute T cytotoxic cell number (CD8+); this is also called the CD4:CD8 ratio. Statistical analysis was performed on cell percentages as initial analyses showed that absolute counts and percentages gave the same results. The statistical test performed was a non-parametric, two-tailed $t$ test with Mann-Whitney. Statistical significance was considered to have been reached when $p \leq 0.05$.

Post hoc power analysis was conducted on the results presented in Tables 2 and 3, both absolute numbers and percentages, using $\mathrm{G}^{*}$ Power 3.1 (Dusseldorf, Germany). The settings were as follows: test family $=t$ tests; statistical test $=$ means Wilcoxon-Mann-Whitney test (two groups); type of power analysis $=$ post hoc compute achieved power given $\alpha$, sample size and effect size. Input parameters were two-tailed, normal parent distribution, $\alpha$ err prob $=0.05$. The effect size was calculated based on the observed means and SDs, and the sample sizes were as listed in the data. Results of this analysis varied by cell type and data sets compared.

\section{Results}

\section{Power analysis}

Assessment of the statistical power of these data sets revealed variation in the power achieved between cell types,

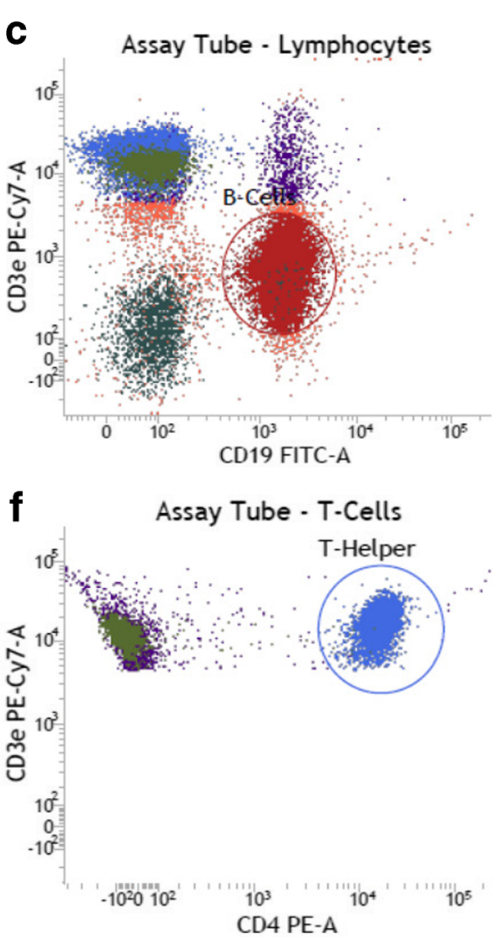

indicating NK cell population based on NK1.1 expression. e Green gate indicating $\mathrm{T}$ cytotoxic cell population based on $\mathrm{CD} 8$ expression. f Blue circle gate indicating $\mathrm{T}$ helper cell population based on $\mathrm{CD} 4$ expression

absolute numbers and percentages as well as in data sets compared. Therefore, different numbers of animals would be required to increase the power depending upon the cell type of interest. As this is not practical, we recommend that each laboratory have an understanding of the power achieved by their study design and potential effects on data interpretation. In general, where statistical significance was achieved, the power was as follows: $*=0.5-.65, * *=$ $0.79-.93$ and $* * *=0.72-1$. As this data is intended for use as background data, the power will change as data is continually added from control animals on our routine studies.

\section{Rat immunophenotyping}

\section{Strain differences}

The data for this analysis is presented in Table 2. At 2 months of age, there are significant differences in the percentage of cells which make up the lymphocyte and $T$ cell subpopulations in $\mathrm{Crl}: \mathrm{WI}(\mathrm{Han})$ vs. $\mathrm{Crl}: \mathrm{CD}(\mathrm{SD})$ rats. These differences occur in both sexes for $\mathrm{B}$ cells, $\mathrm{T}$ helper and $\mathrm{T}$ cytotoxic cells, while there is a statistically significant change in the T cell population for males only. The NK cell population is the only population where there is no apparent significant difference between the strains. 


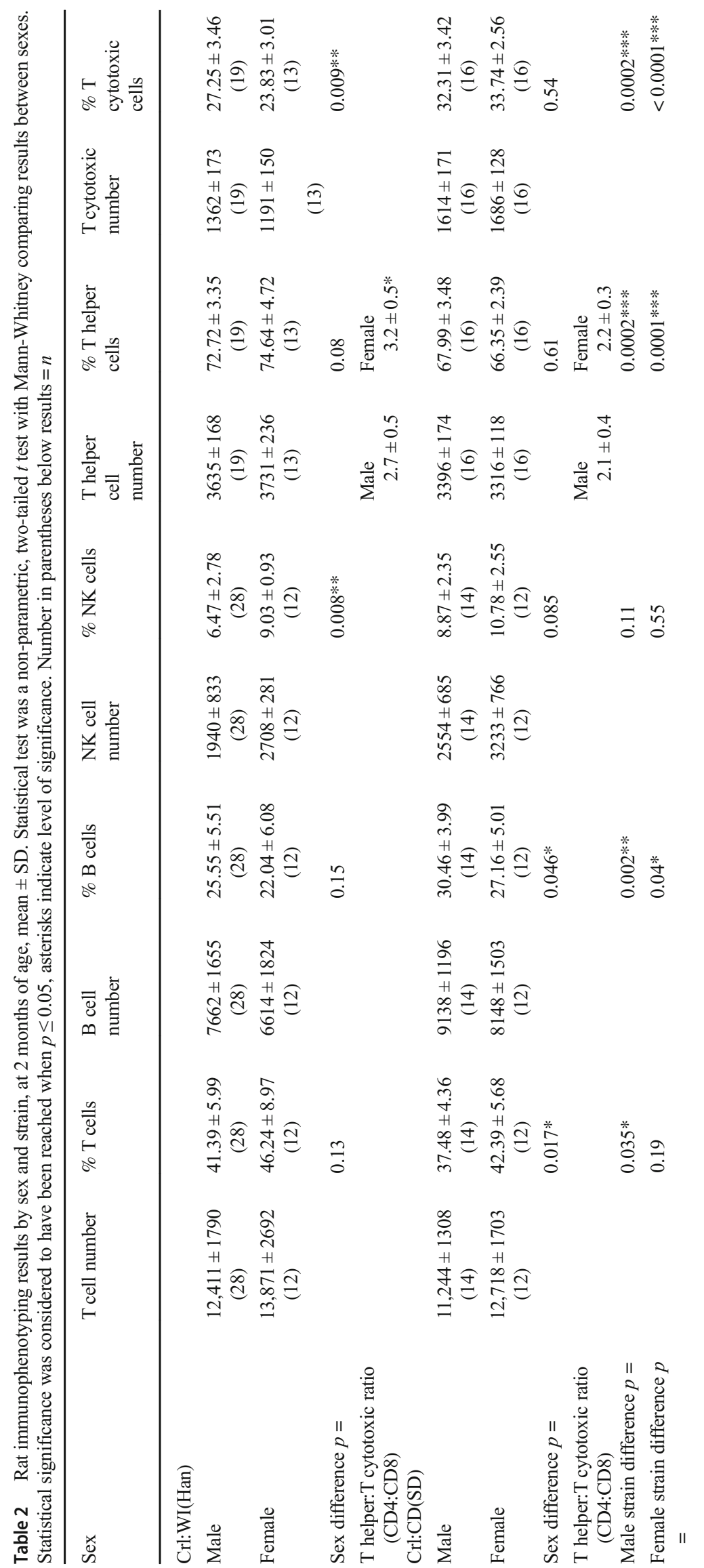




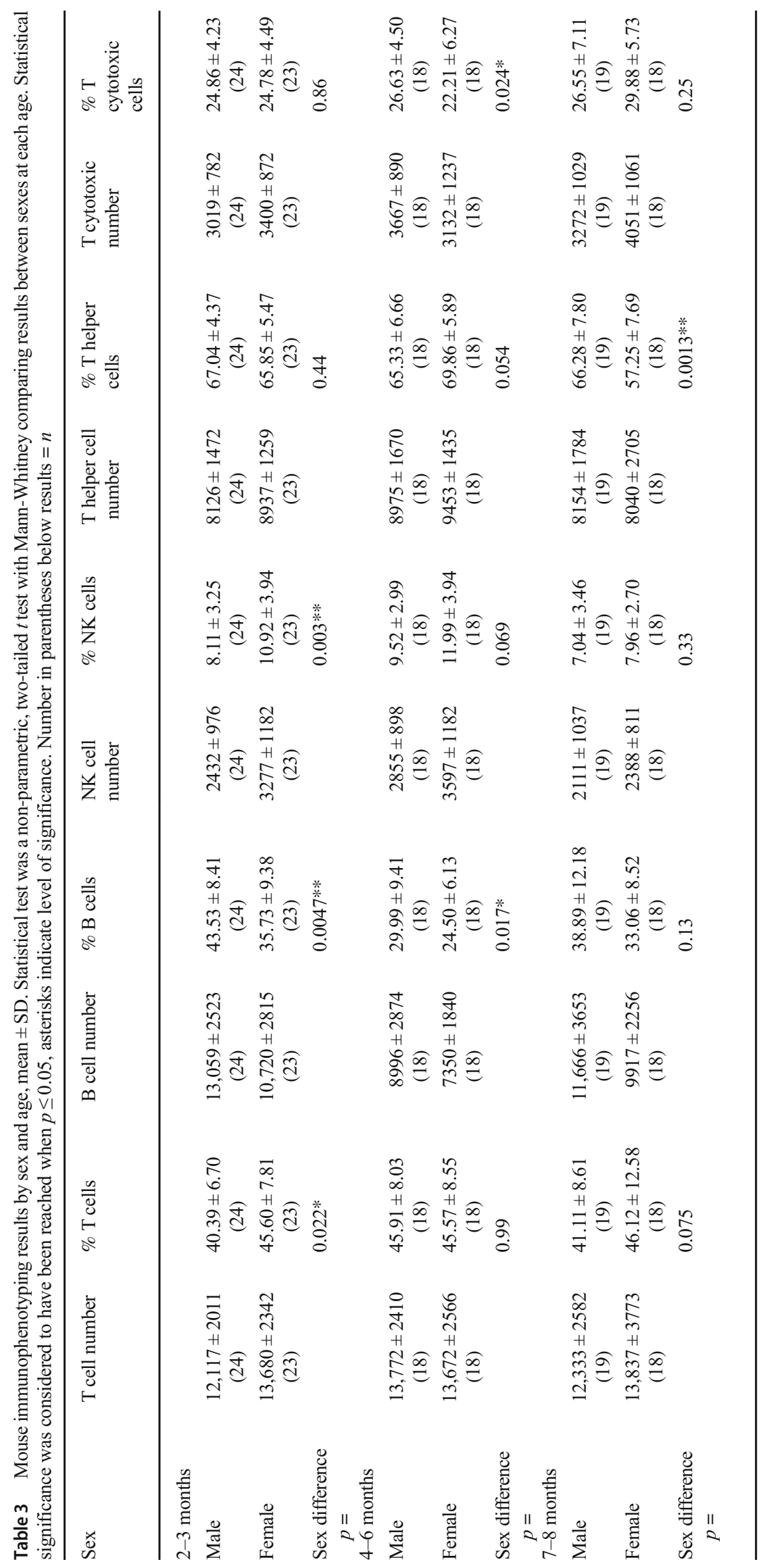




\section{Sex differences}

At 2 months of age, Crl:WI(Han) animals have statistically significant differences in amounts of NK and T cytotoxic cells in their lymphocyte pool depending upon their sex (Table 2). Females show an increased number of NK cells and decreased number of T cytotoxic cells in comparison to males. There is also a statistically significant difference in the $\mathrm{T}$ helper/T cytotoxic $(\mathrm{CD} 4: \mathrm{CD} 8)$ ratio between the sexes in this strain.

At 4 and 7 months of age (Fig. 4), there are statistically significant differences in the percentage of B cells in the lymphocyte pool with females having fewer B cells than males. The only other significant difference is seen in the T cell population at 7 months of age where females have higher numbers of T cells. All other populations show no difference between the sexes at these ages.

At 2 months of age, $\mathrm{Crl}: \mathrm{CD}(\mathrm{SD})$ animals have statistically significant differences in amounts of $\mathrm{T}$ and $\mathrm{B}$ cells in their lymphocyte pool depending upon their sex (Table 2). Females show an increased number of $\mathrm{T}$ cells and decreased number of $\mathrm{B}$ cells in comparison to males.

\section{Age differences}

In the Crl:WI(Han) strain, there were age-related differences in the percentages of cells which make up the lymphocyte pool (Fig. 4). As rats age, there are increases in the number of B cells with decreases in the NK cell numbers. These changes are statistically significant between 2 and 4 or 7 months, but not between 4 and 7 months. T cell populations show a different pattern of change with a decrease in $\mathrm{T}$ cell numbers that is significant between 2 and 7 months or 4 and 7 months in males, but only between 2 and 7 months in females. Interestingly, there are changes in the T cell sub-populations, which do not appear to affect the overall $\mathrm{T}$ cell numbers. Higher numbers of $\mathrm{T}$ helper cells with age reached statistical significance between 2 and 4 or 7 months in males, and between 2 and 7 or 4 and 7 months in females. These changes were accompanied by decreases in the T cytotoxic cell population that were statistically significant at all points tested in the males, and between 2 and 4 or 7 months in females.

\section{Mouse immunophenotyping}

\section{Sex differences}

Data are presented in Table 3 for each age and sex. In Crl:CD1(ICR) mice, it appears that sex differences diminish with age; as by $7-8$ months, the only significant difference is in the number of $\mathrm{T}$ helper cells making up the $\mathrm{T}$ cell pool. At younger ages of either 2-3 or 4-6 months, sex differences are seen in B cells. Statistically significant differences are seen in
T and NK cells only at 2-3 months and in T cytotoxic cells at 4-6 months.

\section{Age differences}

Data are presented in Table 3 and statistical information is contained in Fig. 5. As mice age from 2 to 3 months to 4 6 months, regardless of sex, there is a decrease in the B cell number and increases in the NK cell numbers. Statistical significance is reached for B cells when comparing 2-3 and 46 months. In NK cells, statistical significance is reached when comparing 2-3 and 4-6 months in males but not females. This trend appears reversed at 7-8 months where there is an increase in the B cell number and decrease in the NK cell number. The changes in $\mathrm{B}$ cell numbers for both sexes are statistically significant when comparing 4-6 and 7-8 months, while in NK cells, statistical significance is reached when comparing 4-6 and 7-8 months in males and 2-3 or 4-6 and 7-8 months in females. The $\mathrm{T}$ cell populations appear to be more stable in mice and the only point of statistical significance was an increase in male $\mathrm{T}$ cell numbers when comparing 2-3 and 46 month data. Interestingly, there are changes in the T cell subpopulations in females which appear to be age related and do not appear to affect the overall $\mathrm{T}$ cell numbers. $\mathrm{T}$ helper cell numbers are initially higher at 4-6 months and then fall at $7-$ 8 months. Statistical significance is reached when comparing data from 2 to 3 or 4-6 and 7-8 months. These changes are accompanied by the opposite pattern in the T cytotoxic cell population with the same pattern of statistical significance.

\section{Discussion}

An important component of any assay used within a GLPcompliant laboratory is the ability to perform the assay routinely, with the same parameters over days, weeks, months or even years. It is this reproducibility that allows for the progressive steps of toxicology investigations to show the safety margins of a new treatment or chemical over different dose levels, time periods, sexes and ages. Flow cytometric analysis of peripheral blood cell populations can be an important part of this investigation, particularly where prior knowledge indicates that the immune system may be affected by a test item. The FACSVerse flow cytometer and its associated software, FACSuite, is a useful system for ensuring that analysis is reproducible when the Quality Control functions, tube and assay settings are used in conjunction with Assay Programming. This system is geared towards the standardisation of collection parameters and protocols between users, instruments and laboratories. Assay settings with gating protocols can be exported and shared between laboratories. CS\&T and Research FC beads are used for instrument and assay setup, ensuring that compensation settings are such that collection parameters are 

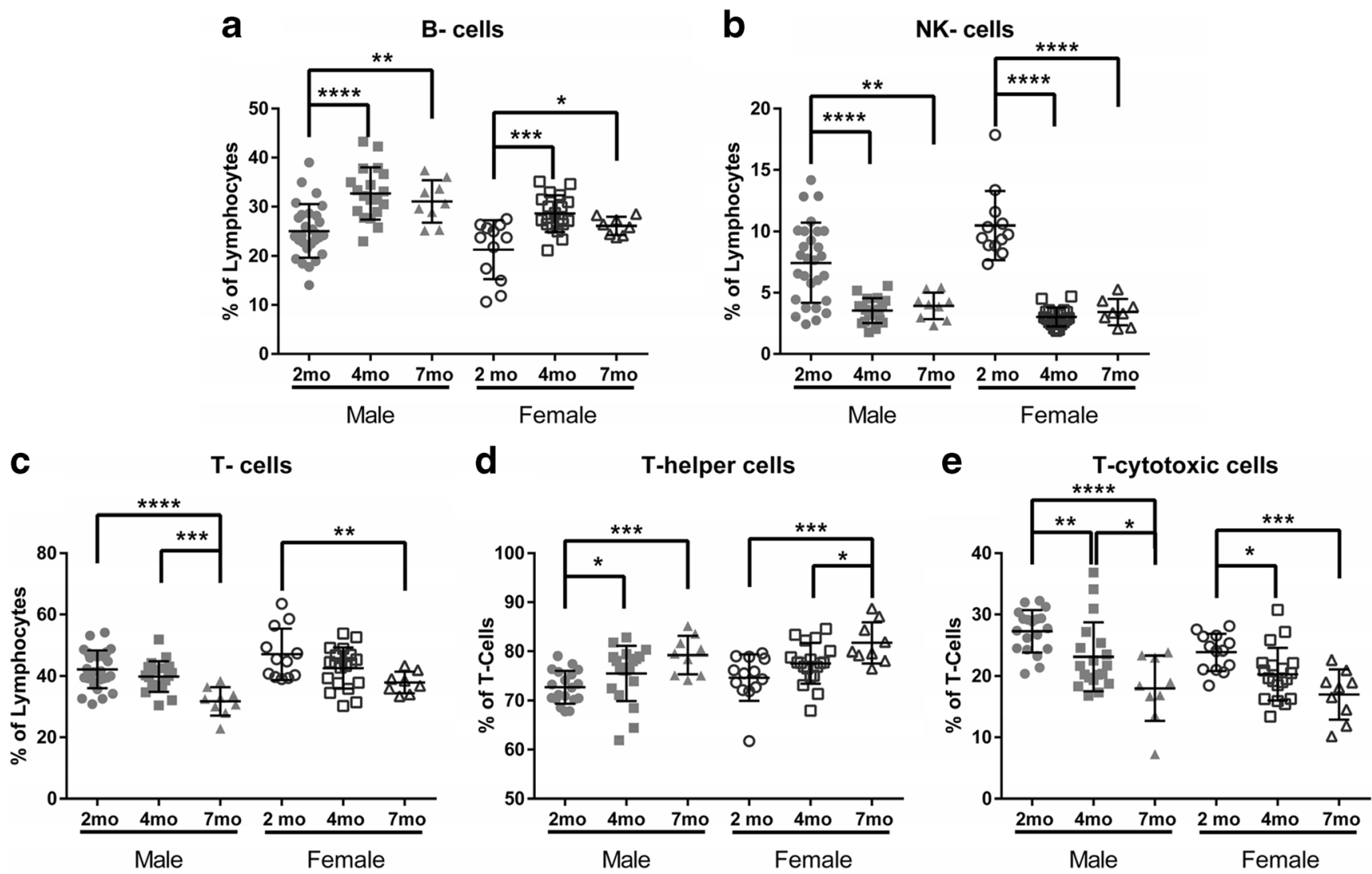

Fig. 4 Immunophenotyping of Crl:WI(Han) rats at 2, 4 and 7 months of age. a B cells. b NK cells. $\mathbf{c} T$ cells. $\mathbf{d}$ T helper cells. e T cytotoxic cells. All results are presented as the percentage of cells making up their parent population (lymphocytes for $\mathrm{B}, \mathrm{NK}$ and T cells, T cells for $\mathrm{T}$ helper and $\mathrm{T}$

cytotoxic cells). Each point is an individual animal. Error bars show the mean \pm SD. Asterisks indicate statistically significant differences between the ages in the same sex, $* p \leq 0.05 ; * * 0.005 ; * * * 0.0005 ; * * * * 0.0001$

the same across time, instruments, laboratories and users. This system also has functions which allow for the electronic approval, annotation and electronic signature of data which contributes to GLP adherence and data security.

Regardless of the instrument and software used, an assay needs to be validated to ensure that it is functioning to an acceptable standard. Validation also ensures that when an assay component is changed, antibody batches, for example, this does not change the function or results of the assay. Validation parameters tested for this assay included intra-assay (withinrun) precision, inter-assay (between-run) precision, sample fixation and stability and staining profile. In this case, samples were not stable enough to make a positive statement on interassay precision. However, intra-assay precision where samples were stained and analysed in triplicate had coefficients of variation of less than $\pm 20 \%$. This showed that the staining and analysis procedure did not introduce variability into samples. The staining profile of each cell type was plotted against the other markers used in the assay to show that there was minimal number of cells which expressed multiple markers and the expression profile of cells was as expected and listed in Table 1. The data from this study is compared against data from control (untreated) animals of a similar age in routine studies so that if there are any changes induced by different batches of antibodies, these can be annotated as such. As an additional control measure, the procedure of performing this analysis has been detailed in a method document which cannot be changed without review and signature of multiple scientists and changes to the assay itself cannot be performed without administrator-level access rights to the FACSuite software. A copy of this method, electronic copies of the assay setup prior to analysis, as well as all FCS, CSV and PDF files are kept with each study so that they may be reviewed as part of Quality Assurance assessments.

The data sets presented in this paper have been collected over multiple analysis days and offer insight with larger numbers of samples compared to other published studies (Morris and Komocsar 1997, Petkova et al. 2008, Pinchuk and Filipov 2008, Yamatoya et al. 2012) which have indicated that sex and strain differences are present in lymphocyte sub-populations. But the effects of age have not been robustly assessed. While it is difficult to compare absolute cell numbers between published studies due to strain, collection and gating differences, it can be noted that the CD4:CD8 ratio obtained in this study for 2-month-old Crl:CD(SD) rats is similar to that published by Morris and Komocsar (1997) where they used Sprague 


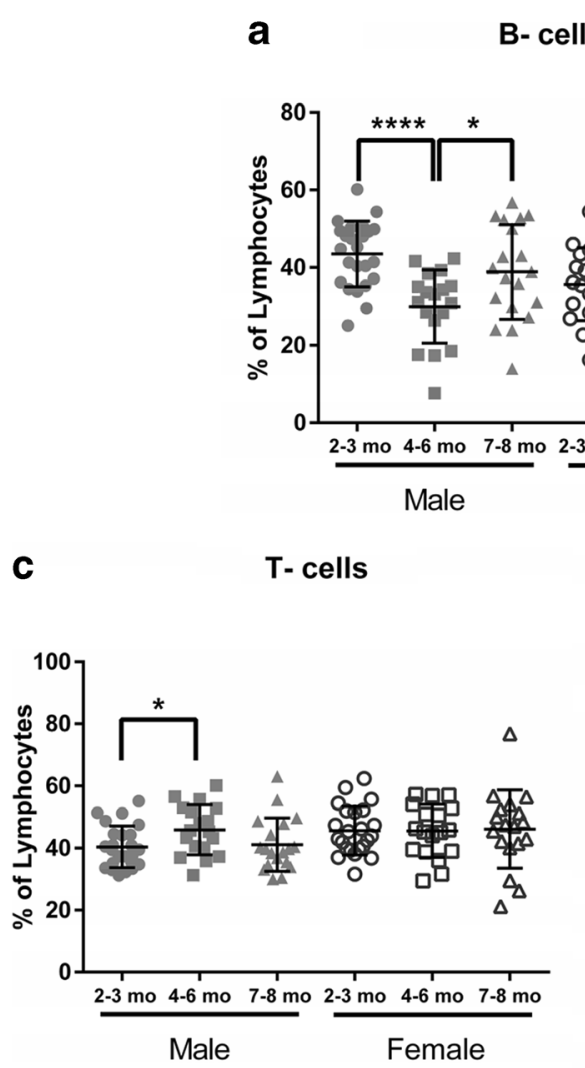

Fig. 5 Immunophenotyping of Crl:CD1(ICR) mice at 2-3, 4-6 and 7-8 months of age. a B cells. b NK cells. c T cells. d T helper cells. e T cytotoxic cells. All results are presented as the percentage of cells making up their parent population (lymphocytes for B, NK and T cells, T cells for

Dawley rats obtained from Charles River in the USA, as are the percentages of $\mathrm{B}$ cells and $\mathrm{CD} 3+\mathrm{T}$ cells. Although as shown here, strain differences can be marked and could exist between different suppliers of Sprague Dawley rats. Therefore, it is important that when published, there is specific strain information so that comparability between studies can be assured.

The data collected here demonstrate that there is potential for standard total lymphocyte counts to be overly simplistic where there is potential for effects on the immune system. For example, in the Crl:CD1(ICR) mouse data set, T cell numbers do not vary widely across either sex or age; however, when the sub-populations of $\mathrm{T}$ helper and $\mathrm{T}$ cytotoxic cells are investigated, females show highly significant differences in the ratios of cells that make up their $\mathrm{T}$ cell population as they age. Similarly, in the same cell types in rats, although the changes appear small (differences of 5-10\%), they are highly statistically significant, particularly between the strains. Therefore, discrimination of the counts or relative percentages of each sub-population could indicate an aspect of toxicity or potential mechanisms of action not highlighted by the total lymphocyte or even T cell count, which may not change. It is also possible that due under powering indicated in some parameters, particularly $\mathrm{T}$ cells, there may be differences which have not been

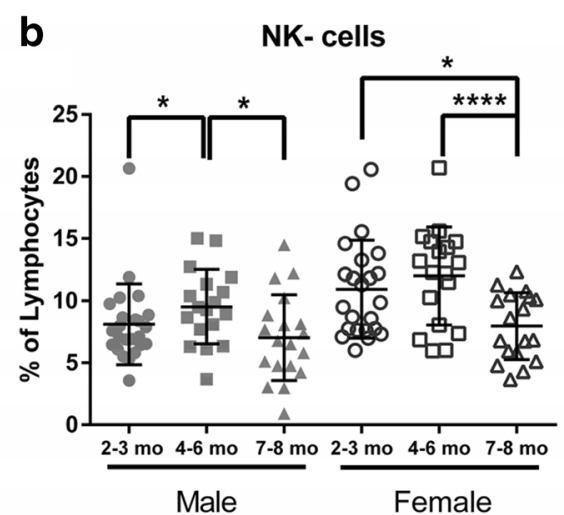

T-helper cells

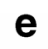

e

e T-cytotoxic cells

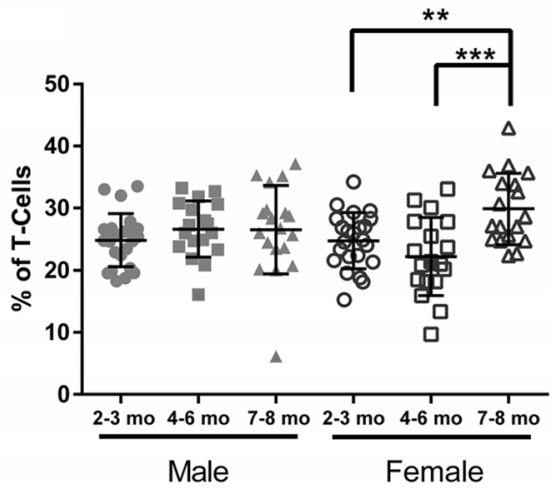

Female

T helper and T cytotoxic cells). Each point is an individual animal. Error bars show the mean \pm SD. Asterisks indicate statistically significant differences between the ages in the same sex, ${ }^{*} p \leq 0.05 ; * * 0.005$; $* * * 0.0005 ; * * * * 0.0001$

detected in these data sets. However, as this data is intended for use as background data, the power will change as data is continually added from control animals on our routine studies, and so may improve. Also, where statistical significance was achieved, the higher the significance the higher the power indicated, so while there may be some differences which are not yet distinguishable, the differences currently seen are robust.

In terms of toxicology investigations, generally, both absolute cell numbers and percentages are used to evaluate any effects which may be induced by test articles. But, where numbers are limited by stopping criteria, critical thought on the scale and direction of any changes or differences is needed. It should be considered if an increase in one cell type results in a decrease in another cell type or vice versa, it should be kept in mind that this change may not be wholly reflective of the cell number in the circulation. There is also the possibility that the cell types interrogated may miss a cell type, and therefore, decreases in the lymphocyte subsets counted may be due to an increase in a cell type which does not have a specific lymphocyte sub-gate in the protocol. As an example, there is a subset of T cells, natural killer T cells, which may not express either CD4 or CD8, but increase in some inflammatory situations (RnD systems accessed 2018). If such a response was assayed 
by the methods in this paper, T cell number may be as expected, but CD4 and CD8 populations could be decreased.

It is critical that immunophenotyping data be considered in conjunction with routine haematology data. In the assays presented in this paper, lymphocyte numbers are normalised for staining purposes, but the initial absolute count of lymphocytes needs to be considered in relation to the WBC count. It is possible that 30,000 lymphocytes can still be counted even when there are reductions to this population in the haematology analysis. As an alternative, assays could be setup to count cells for a fixed amount of time, so that any changes in the absolute lymphocyte number may be detected and accounted for in the assay. Whichever method is used needs to be clearly stated with the results so that accurate interpretation can be applied, as it is possible to get a statistical difference in either absolute numbers or percentages and not both which would change the way that the data is interpreted. Additionally, power analysis can be conducted to ensure that the data set is of a size sufficient to accurately assess the differences in study groups. Although it should be noted that the number of animals needed may vary by cell type of interest due to the different variability in the individual cell types, or dictated by guidelines associated with the study type.

The use of both sexes in investigations, which may affect lymphocyte subsets, is an important part of study design. Depending upon the guidelines relevant for the study, OECD, EPA, FDA, etc., it may be possible to use the more sensitive sex, where this is known. Therefore, when designing a study, it is important to consider the utility of using both sexes considering any previously established data. As shown in this study, while the significant differences are not universal to a single cell type, there are sex differences in at least one cell type of each species, strain and age examined. However, it should be noted that in mice at least, the sexes are the most similar in animals of 7-8 months of age. Therefore, if a background database for T, B and NK cell data is produced using a single sex at this age, it could contribute to the principles of the 3Rs and be used as a baseline for comparison against both sexes.

As with any investigation of this nature, the robustness of the results is hampered by the relatively small sample size. Although the number of samples investigated here is substantially greater than has been reported elsewhere, it is as yet unclear whether some of the differences highlighted represent a true difference, or whether there is quite substantial variation. This will only become clear as a greater volume of data is gathered, and we would encourage all those working in this area to share data, to either confirm the changes we have seen as true age- and sex-related variances, or alternatively, to clarify expected ranges for laboratory animals.

\section{Compliance with ethical standards}

Ethical approval All applicable international, national and institutional guidelines for the care and use of animals were followed. All procedures performed in studies involving animals were in accordance with the ethical standards of the institution at which the studies were conducted.

Conflict of interest The authors declare that they have no conflict of interest.

Open Access This article is distributed under the terms of the Creative Commons Attribution 4.0 International License (http:// creativecommons.org/licenses/by/4.0/), which permits unrestricted use, distribution, and reproduction in any medium, provided you give appropriate credit to the original author(s) and the source, provide a link to the Creative Commons license, and indicate if changes were made.

\section{References}

Green CL, Brown L, Stewart JJ, Xu Y, Litwin V, McCloskey TW (2011) Recommendations for the validation of flow cytometric testing during drug development: I instrumentation. J Immunol Methods 363: 104-119

International conference on harmonisation of technical requirements for registration of pharmaceuticals for human use (2005) ICH harmonised tripartite guideline immunotoxicity studies for human pharmaceuticals, S8. http://www.ich.org/fileadmin/Public_Web Site/ICH_Products/Guidelines/Safety/S8/Step4/S8_Guideline.pdf

Morris DL, Komocsar WJ (1997) Immunophenotyping analysis of peripheral blood, splenic, and thymic lymphocytes in male and female rats. J Pharmacol Toxicol Methods 37:37-46

O'Hara DM, Xu Y, Liang Z, Reddy MP, Wu DY, Litwin V (2011) Recommendations for the validation of flow cytometric testing during drug development: II assays. J Immunol Methods 363:120-134

Petkova SB, Yuan R, Tsaih SW, Schott W, Roopenian DC, Paigen B (2008) Genetic influence on immune phenotype revealed strainspecific variations in peripheral blood lineages. Physiol Genomics 34:304-314

Pinchuk LM, Filipov NM (2008) Differential effects of age on circulating and splenic leukocyte populations in C57BL/6 and BALB/c male mice. Immunol Aging 5:1. https://doi.org/10.1186/1742-4933-5-1

RnD Systems, https://www.rndsystems.com/research-area/natural-killert-nkt-cells, Accessed March 2018

Yamatoya H, Kawaguchi H, Fukuda T, Kadokura H, Yamashita R, Yoshikawa T, Shiraishi M, Miyamoto A, Miyoshi N (2012) Data on Wistar Hannover rats from an immunotoxicity study. Exp Anim 61:171-175 